\title{
Dimensional interactions and the structure of psychological space: The representation of hue, saturation, and brightness
}

\author{
BARBARA BURNS \\ Mount Holyoke College, South Hadley, Massachusetts \\ and \\ BRYAN E. SHEPP \\ Brown University, Providence, Rhode Island
}

\begin{abstract}
The perception of color has traditionally been characterized by the subjective dimensions of hue, brightness, and saturation. In the present study we reexamined this view by investigating whether the dimensions of color stimuli are psychologically independent in dissimilarity judgment, spontaneous classification, and instructed classification tasks. Dissimilarity judgments analyzed within the framework of the additive difference measurement model (Beals, Krantz, \& Tversky, 1968; Krantz \& Tversky, 1975; Tversky \& Krantz, 1969, 1970) reflected violations of psychological independence for hue-chroma, hue-value, and value-chroma stimulus sets. Spontaneous classifications of each color set revealed that subjects were not sensitive to shared dimensional relations of color stimuli, but rather responded to the holistic, overall similarity relations of the stimuli. In the instructed classification task, both untutored undergraduates and "color experts" (artists specially tutored in the Munsell color system), instructed to classify according to shared dimensional relations, could extract dimensional information about either value or chroma when each was varied with hue, but could not extract dimensional information about hue. Color experts were superior to nonexperts in the extraction of dimensional information about chroma only with moderately or highly saturated stimuli. The implications of these results are considered in relation to current thinking about the perceptual organization of color and current thinking about the identification of appropriate diagnostics for independent psychological dimensions.
\end{abstract}

The precise notion of a psychological dimension continues to elude psychologists interested in the perception and organization of multidimensional stimuli. Defining a psychological dimension has been particularly problematic for investigators interested in color. A common assumption has been that the perception of color is organized in terms of the dimensions hue, brightness, and saturation. "There is no doubt, in the case of color differences, as to the number of independent perceptual attributes... The number is three" (Krantz, 1972, p. 683). This assumption is based on scaling procedures (i.e., confusability scaling, direct estimation, multidimensional scaling, etc.) that have supported the idea that hue, brightness, and saturation are the dimensions of color space (Helm, 1964; Indow \& Kanazawa, 1960; Indow \& Uchizono, 1960; Newhall, 1939; Newhall, Nickerson, \& Judd, 1943; Wright, 1965).

This research was supported by National Institute of Child Health and Human Development Grant HD-11915, a faculty fellowship from Mount Holyoke College to the first author, and Grant HD-13406 to the second author. We are grateful to Chris Ho, Karen Harris, Martha Smith, Frank Slater, and Willa Wiener-Ehrlich for their help in data collection and analysis. Requests for reprints should be sent to Barbara Burns, Department of Psychology, University of Louisville, Louisville, KY 40292.
One difficulty with this assumption that hue, brightness, and saturation are the three dimensions organizing the perception of color is related to the multiple uses of the term dimension. As Tversky and Krantz (1970) and Gati and Tversky (1982) indicated, the term has been given several meanings in the literature. Psychologists have used the term dimension simply to refer to a variable that can be physically manipulated. The term has also been used to mean an organizing principle that structures and orders perception in a consistent way. A third use of the term stipulates that the organizing principle of a particular set of perceptual experiences is psychologically independent of any other set.

The major question in the present research focuses on this third definition of dimension. We ask whether the dimensions, or organizing principles, of color stimuli are, in fact, psychologically independent. There is considerable evidence that the physical attributes of color do not independently affect the psychological dimensions of color. For example, variation on a single physical attribute of color produces variations on more than one subjective dimension (e.g., the Bezold-Brücke effect). (See Boynton \& Gordon, 1965, and Yager \& Taylor, 1970, for reviews.) We are concerned with whether the attrib- 
utes of color are perceptually independent, and especially with the conditions under which independence does or does not exist.

In studies on dimensional interactions, Garner (1970, $1974,1976)$ and Lockhead $(1972,1979)$ formalized distinctions initially made by Shepard (1964), who argued that different dimensions combine to yield fundamentally different percepts. Stimuli that are generated by combining "separable" dimensions, such as size of circle versus angle of a radial line, are perceived as conjunctions of distinct features or values. In contrast, stimuli that are generated by combining "integral" dimensions, such as height versus width of a rectangle, are not perceived as conjunctions of distinct features; they are perceived in terms of more holistic properties of the stimulus object.

Perceived differences in structure have yielded very different performances in a variety of converging tasks. Separable dimensions yield a city-block metric in direct distance scaling, do not give a redundancy gain in speeded tasks with correlated values, and allow selective attention to one dimension when a second is varied orthogonally. Integral dimensions, on the other hand, are characterized by a Euclidean metric in direct distance scaling, produce a redundancy gain in speed or accuracy with correlated values, and do not allow selective attention to one dimension when a second is varied orthogonally (Garner, 1974, 1976).

The work on dimensional interactions indicates that subjects respond to holistic similarity relations among color stimuli and not to dimensional relations (Garner, 1974; Smith \& Kemler, 1978). Although this evidence contradicts the traditional view that color is perceived according to hue, brightness, and saturation, it does not rule out the possibility that the perception of shared dimensional relations (which underlies the diagnostics of dimensional independence) in color stimuli is possible. Both Lockhead (1972) and Garner (1974) took the view that subjects may respond to either the holistic or the dimensional relations among integral stimuli. Lockhead argued that integral stimuli are first perceived as "blobs" and subsequently are analyzed into dimensional features, but only if the demands of the task require analysis of the stimuli. In a similar vein, Garner distinguished between primary and secondary processes in the perception of multidimensional stimuli. The primacy process occurs first and is automatic; the secondary process occurs second and is the result of more cognitive operations. According to Garner, the primary process for all integral stimuli detects overall similarity relations among stimuli, whereas the secondary cognitive process detects dimensional relations.

The argument by Garner (1974) and Lockhead (1972) that all dimensional combinations can be analyzed (although for integral dimensions, not automatically) identifies a fundamental property of stimulus dimensions, namely, that their respective features must be subjectively independent. Despite the fundamental nature of subjective independence, it has seldom been tested, and only recently have some formal criteria for subjective independence been provided.
Beals, Krantz, and Tversky (1968), Tversky and Krantz $(1969,1970)$, and Krantz and Tversky (1975) developed a quantitative psychological model that specifies defining properties for subjectively independent dimensions. The model, referred to as the additive difference measurement model (ADMM), provides testable axioms that specify whether one attribute (e.g., hue) is psychologically independent relative to another (e.g., brightness).

The most frequent tests of the ADMM have examined one aspect of the axiom of interdimensional additivity, namely, the property of equality. Consider the stimulus set illustrated in Figure 1. To test the equality prediction, a given interval on one dimension is examined at several constant levels on a second dimension. For example, the dissimilarity ratings of stimulus pair $(1,5)$, which differ by one interval on dimension $Y$, would be compared with the ratings of pairs $(2,6),(3,7)$, and $(4,8)$. If dimensions $X$ and $Y$ are independent, then each of these stimulus pairs is judged according to their interval difference on dimension $\mathrm{Y}$; the constant value of $\mathrm{X}$ should not contribute differentially to the judgment of any pair. Thus, the ratings of the four pairs should be equal. Similarly, the remaining one-step, two-step, and three-step unidimensional intervals could be compared. A second part of the equality test involves comparisons of stimulus pairs that differ by the same interval on each dimension. For example, in Figure 1 the stimulus pairs $(1,6)$ and $(2,5)$ differ by one interval on each dimension. The stimulus pairs $(1,10)$ and $(2,9)$ differ by one interval on dimension $X$ and two intervals on dimension $Y$. These bidimensional variations can be compared to determine whether dissimilarities on dimensions are combined equally.

The experimental evidence shows that some dimensional combinations meet this test of perceptual independence, and others do not. Tversky and Krantz (1969) showed that dissimilarity judgments of schematic faces (e.g., depth of frown, eyebrows) exhibited equality. Wender (1971) investigated the same axioms of equality, using rating scale judgments of rectangles that varied

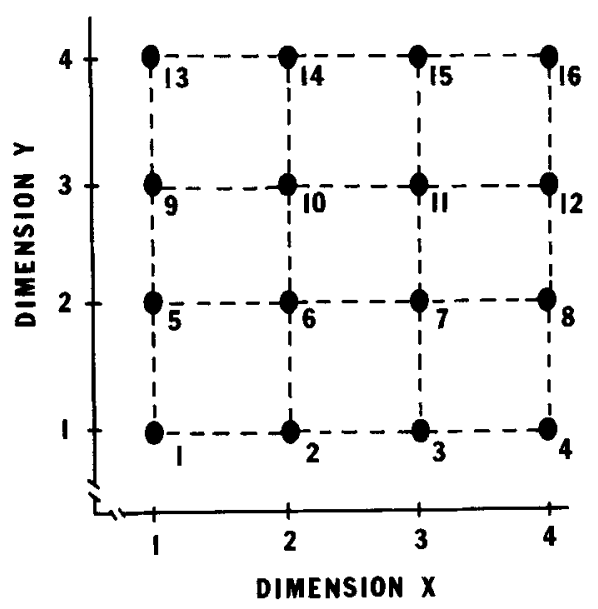

Figure 1. A $4 \times 4$ stimulus set used to portray the critical relations of interdimensional additivity. 
in area and shape, and found significant violations of equality.

Several investigators (Burns, 1976; Burns, Shepp, McDonough, \& Wiener-Ehrlich, 1978; Wiener-Ehrlich, 1978) have argued that the perceptual independence of the ADMM should agree with the converging operations provided by Garner (1974) to distinguish integral and separable stimuli. Integral stimuli are perceived holistically; stimuli that are integral by Garner's converging tasks should violate additive difference metrics. Separable stimuli are perceived as independent component dimensions; stimuli that are separable should meet the assumptions of additive difference metrics.

Burns et al. (1978) provided tests of this argument by comparing proximity data and patterns of performance in tasks that have been used as diagnostic for integral and separable stimuli within Garner's (1974) framework. Their results showed close agreement between Garner's operational definitions of integrality and separability and the acceptance and failure of the predictions from the ADMM. The dimensions of separable stimuli (size vs. brightness, circle vs. angle) consistently met the requirements of interdimensional additivity. That is, in the rating scale data, the dissimilarity between two separable stimuli was composed of the independent contributions along each constituent dimension. Similarly, in the classification paradigm, subjects consistently based judgments on shared identity on one dimension rather than on a holistic combination of similarities across all dimensions. In contrast, the dimensions of integral stimuli (area vs. shape, height vs. width, hue vs. chroma) did not meet the ADMM's requirements for psychological independence, and classifications were based predominantly on overall similarity.

The present experiments extended this analysis of the ADMM and Garner's (1974) framework in two important ways. First, do all sets of integral stimuli interact and show the pattern of results reported by Burns et al. (1978)? In Experiments 1 and 2 we investigated the perceptual organization of each of the possible color combinations-hue versus saturation, hue versus brightness, and brightness versus saturation-using both dissimilarity judgment and restricted classification tasks. Second, do the interactions between the attributes of color reflect only a primary response to color? Is there a secondary, analytical response, based on independent features, that can be tapped by instruction or special experience? In Experiments 3 and 4 we addressed these questions.

\section{EXPERIMENT 1}

In Experiment 1, subjects made dissimilarity judgments about three sets of color stimuli: hue-chroma, hue-value, and chroma-value. Previous research had shown that when the features in multidimensional stimuli were obvious, then the features of one dimension were judged independently from features of a second dimension. When features were not obvious, judgments of independence fail (Krantz \& Tversky, 1975; Tversky \& Krantz, 1969, 1970).

Our previous research (Burns et al., 1978) had shown that hue and chroma were not independent, and in the present experiment we extended the analysis to the other combinations of color attributes. There was no previous evidence that one combination of color attributes was any more likely than another to show perceptual independence. It is important to note, however, that distinctions between integral and separable dimensions refer to interactions between combinations of two or more dimensions. A particular dimension, such as brightness, may be separable relative to size but integral relative to saturation (e.g., Handel \& Imai, 1972). Thus, any general statements about color perception require an analysis of all possible combinations.

\section{Method}

Subjects. Thirty subjects were recruited from the Brown University undergraduate subject pool. Ten subjects were assigned to judge each of the three stimulus sets, with approximately equal numbers of males and females assigned to each set. Prior to the experimental task each subject was administered the Ishihara test for colorvision deficiency. Subjects were paid $\$ 3$ for each of two sessions.

Stimuli. Three stimulus sets were constructed for use in all four experiments, using Munsell glossy chips that varied in hue, value (brightness), and chroma (saturation). The chips were presented as $2.5-\mathrm{cm}$ squares mounted on gray (Munsell value $=5$ ) square cards that measured $11.5 \mathrm{~cm}$ on a side. In all experiments the stimuli were viewed under a lamp provided by the Munsell Company to approximate illuminant $\mathrm{C}$. The hue-chroma set was composed of 36 stimuli that varied in six levels of hue (10RP, 2.5 R, 5R, 7.5 R, $10 R$, and $2.5 Y R)$ and six levels of chroma $(2,4,6,8,10$, and 12). All stimuli in the hue-chroma set had a value level of 6 . The hue-value set consisted of 36 stimuli that varied in six levels of hue (10RP, 2.5R, 5R, 7.5R, 10R, and 2.5 YR) and six levels of value $(3,4,5,6,7$, and 8$)$. The stimuli in the hue-value set had a chroma level of 12 . The value-chroma set consisted of 36 stimuli that varied in six levels of value $(3,4,5,6,7$, and 8$)$ and six levels of chroma $(2,4,6,8,10$, and 12). Each of these stimuli had a constant hue level of 7.5R. In Experiment 1, a $4 \times 4$ matrix of stimuli was selected from the center of the total $6 \times 6$ matrix (i.e., Levels 2 , $3,4,5$ on each dimension) for each of the three stimulus sets.

Procedure. The subjects were asked to perform a dissimilarity judgment task. On each trial the subjects were presented with a pair of stimuli and were asked to rate the dissimilarity of each pair on a 10-point scale. In each of two experimental sessions the subjects rated a total of 120 pairs of stimuli, consisting of all possible combinations with the exception of identical stimuli. The order of presentation was randomized and a different random order was used for each subject. Additional procedural details for this methodology are given in Burns et al. (1978).

\section{Results and Discussion}

The dissimilarity judgment data for each of the stimulus sets were analyzed in several ways. First, the data were submitted to Kruskal's (1964) MDSCAL IV (Version 5MS). In this way, the best fitting $n$-dimensional scaling solution was determined from the average dissimilarity ratings. The best-fitting solutions for each of the three stimulus sets in the present experiment were two- 
dimensional. The solutions for a Euclidean metric produced stress values of $.059, .047$, and .059 for hue-chroma, hue-value, and chroma-value, respectively. In addition, for each stimulus set, correlations were obtained between the physical values on the Munsell scale and the dimensions yielded by the scaling solutions. For the hue-chroma set the Pearson product-moment correlations were .92 and .96 for hue and chroma, respectively. The correlations for the hue-value set were .68 and .82 for hue and value, whereas the correlations for the value-chroma set were .79 and .65 for value and chroma.

One of the ADMM's tests of interdimensional additivity, the equality prediction, was also employed on each set of dissimilarity judgment data. To test the equality prediction, intervals on one dimension were examined at all constant levels on the second dimension in order to determine whether dissimilarity remained constant. For each dimension there were 36 possible comparisons that composed this test of unidimensional equality. A second part of the equality test involved comparisons of stimulus pairs that differed by the same interval on both dimensions. In the bidimensional equality test these pairs were compared to determine whether dissimilarity was judged equally. A total of 72 comparisons composed this test of equality among dissimilarity judgments.

Hue-chroma. Figure 2 (top panel) shows the scaling solution for the $4 \times 4$ matrix of stimuli varying in hue and chroma presented for dissimilarity judgments. A comparison of Figures 1 and 2 shows that the orderings among the stimuli correspond to the orderings of the Munsell system. The relations among the spaces between the numbered stimuli, however, suggest a dimensional interaction. As shown in Figure 2, with increasing levels of chroma, the dissimilarity judgments for equal levels of hue appear to increase. Table 1 presents the actual number of unidimensional equality violations for each subject's dissimilarity judgments. All 10 subjects evidenced a pattern of dissimilarity judgments such that as the constant level of chroma increased from 4 to 10 , dissimilarity judgments of equal hue intervals increased, an effect that is significant by a binomial test $(p<.01)$. In contrast, dissimilarity judgments of equal chroma intervals at constant levels of hue did not differ significantly $(p>.05)$.

The number of bidimensional equality violations for each subject's judgments is shown in Table 2 . The bidimensional dissimilarity judgments of 8 of the 10 subjects showed consistent equality violations. This is, however, not significant using the binomial test $(p>.05)$. The lack of significance may be accounted for by considering that equality violations reached significance for unidimensional judgments of hue only.

Hue-value. The middle panel of Figure 2 shows the best-fitting scale solution for the stimulus set varying in levels of hue and value. A comparison of the matrices in Figures 1 and 2 reveals that the scaling solution shows an order that is close to the ordering of the Munsell system. The spacings among the ordered stimuli also rev-
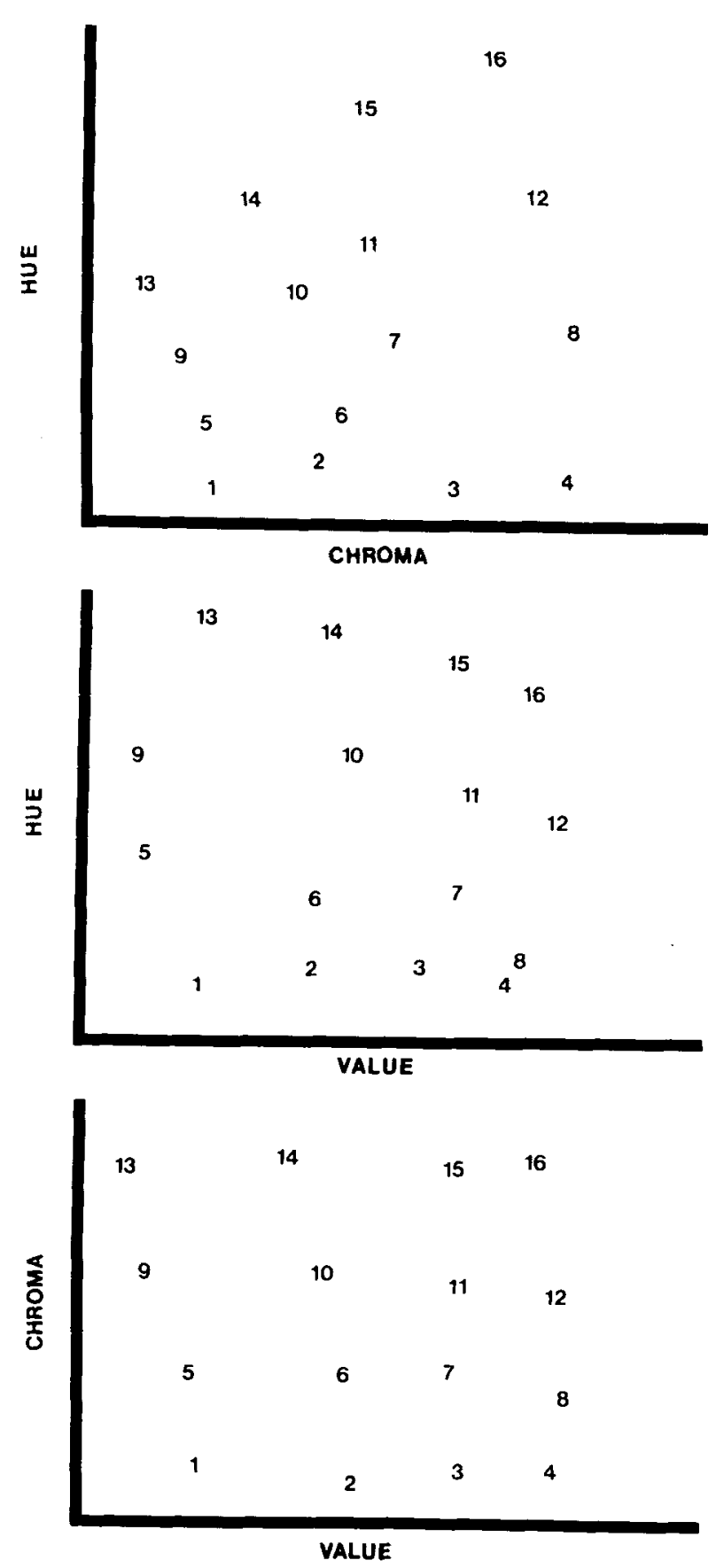

Figure 2. Multidimensional scaling configurations of 16 stimuli in each of three combinations of the Munsell dimensions.

ealed some consistent variations. The same intervals of hue appear to be judged as less dissimilar with increasing levels of value. [For example, compare the distance between 1 and 5 in the pair $(1,5)$ and between 4 and 8 in the pair $(4,8)$.] Table 1 provides the statistical support for this observation; the judgments of 9 of the 10 subjects showed consistent equality violations for the hue intervals $(p<.025)$. Judgments of the unidimensional var- 
iations of value also showed a consistent pattern of equality violations. Nine of the 10 subjects $(p<.025)$ consistently rated intervals of value as more dissimilar when presented with intermediate constant levels of hue than when presented with either of the extremes.

Value-chroma. The stimuli composing the valuechroma set also yielded a well-ordered two-dimensional organization, as shown in the bottom panel of Figure 2 . Table 1 presents the number of equality violations for the dissimilarity pairs in which only one dimension was varied. The same intervals of value were judged as more dissimilar with increasing constant levels of chroma by 9 of the 10 subjects $(p<.025)$. An analogous effect was evidenced for judgments of equal intervals of chroma. Nine of the 10 subjects rated equal intervals of chroma as more dissimilar with increasing levels of constant value. Table 2 presents the number of equality violations for bidimensional variations. Stimulus pairs on the primary diagonal were consistently judged as less dissimilar than equivalent stimulus pairs on the secondary diagonal by 9 of the 10 subjects $(p<.025)$. The dimensional interaction for value and chroma is highly consistent for both unidimensional and bidimensional variations.

Summary. The results of the present experiment support our line of inquiry concerning the perceived organization of color stimuli. On the basis of conventional scaling analyses, one would conclude that an acceptable dimensional structure defines each stimulus set. At stress values of $.059, .047$, and .059 for hue-chroma, hue-value, and chroma-value, respectively, the correlations between the factors of the scaling solutions and Munsell dimensions are significant. The correlations, however, appear to reflect the rank orders of the stimuli and are not sensitive to violations of independence. The visual configurations of the scaling solutions do not reflect independent psychological dimensions. There were significant violations, in varying patterns, of the equality predic- tion derived from the interdimensional additivity axiom of the ADMM for each of the sets of color stimuli.

\section{EXPERIMENT 2}

Although the results of Experiment 1 showed that stimulus sets generated by dimensional combinations of hue, value, and chroma were not perceived in terms of independent psychological constituent dimensions, it was possible that such results were task dependent. Experiment 2 was intended to converge on the characterization of perceived color structure by employing a second type of task which assessed the subjects' perception of shared dimensional relations among stimuli.

In the restricted classification task, triads of stimuli are presented which place two different types of classification in conflict. The distance relations among the three stimuli in such a classification task are typically presented as in triad Types 1 and 2 in Figure 3. On triads composed of stimuli in these distance relations, classification on the basis of a shared value on one dimension (the $A+B$ "dimensional" classification) is pitted against classification on the basis of overall similarity on both dimensions (the $\mathrm{B}+\mathrm{C}$ "similarity" classification).

In order to assess the effects on color stimuli of varying the salience of perceived dimensional relations, we presented several additional types of triads with differing distance relations. Triad Types 1, 2, 3, and 4 in Figure 3 illustrate four triad variations in which the dimensional classification is pitted against the overall similarity classification. The critical difference across these triad types is how similar stimuli B and C are to each other. Previous studies have shown that as the dissimilarity of stimuli B and C is increased (compare especially Types 2,3 , and 4 ), the number of classifications based on shared dimensional relations increases (Burns, 1986; Burns et al., 1978; Shepp, Burns, \& McDonough, 1980).

Table 1

Equality Violations for Dissimilarity Judgments of Pairs of Munsell Stimuli

\begin{tabular}{|c|c|c|c|c|c|c|c|c|c|c|c|c|}
\hline \multirow[b]{3}{*}{ Subject* } & \multicolumn{4}{|c|}{ Stimulus Set } & \multicolumn{4}{|c|}{ Stimulus Set } & \multicolumn{4}{|c|}{ Stimulus Set } \\
\hline & \multicolumn{2}{|c|}{ Hue } & \multicolumn{2}{|c|}{ Chroma } & \multicolumn{2}{|c|}{ Hue } & \multicolumn{2}{|c|}{ Value } & \multicolumn{2}{|c|}{ Value } & \multicolumn{2}{|c|}{ Chroma } \\
\hline & + & - & + & - & + & - & + & - & + & - & + & - \\
\hline 1 & 0 & 27 & 12 & 13 & 23 & 3 & 5 & 16 & 10 & 22 & 6 & 13 \\
\hline 2 & 4 & 26 & 16 & 10 & 13 & 8 & 8 & 11 & 9 & 18 & 7 & 20 \\
\hline 3 & 4 & 21 & 3 & 24 & 15 & 6 & 5 & 21 & 9 & 19 & 8 & 18 \\
\hline 4 & 3 & 29 & 8 & 14 & 20 & 10 & 5 & 21 & 3 & 17 & 3 & 11 \\
\hline 5 & 0 & 30 & 4 & 21 & 7 & 13 & 6 & 17 & 9 & 13 & 7 & 23 \\
\hline 6 & 2 & 28 & 12 & 12 & 17 & 8 & 6 & 22 & 2 & 17 & 10 & 12 \\
\hline 7 & 1 & 26 & 10 & 19 & 24 & 5 & 2 & 17 & 3 & 24 & 15 & 17 \\
\hline 8 & 3 & 25 & 10 & 10 & 14 & 7 & 6 & 17 & 2 & 27 & 21 & 9 \\
\hline 9 & 3 & 24 & 15 & 11 & 14 & 8 & 10 & 8 & 18 & 13 & 15 & 17 \\
\hline 10 & 0 & 29 & 9 & 24 & 17 & 5 & 11 & 16 & 9 & 23 & 6 & 21 \\
\hline
\end{tabular}

Note-Entries represent the number of times the first stimulus pair of a specific interval [e.g., $(1,5)$; see Figure 1] was judged as more $(+)$ or less $(-)$ dissimilar than consecutive theoretically equal pairs [e.g., $(2,6),(3,7),(4,8)] . N=36$ comparisons. Unequal $N$ s are due to stimulus pairs that were judged as equally dissimilar. *Ten different subjects were assigned to each stimulus set. 
Table 2

Equality Violations for Bidimensional Dissimilarity Judgments of Pairs of Munsell Stimuli

\begin{tabular}{|c|c|c|c|c|c|c|}
\hline \multirow[b]{3}{*}{ Subject* } & \multicolumn{2}{|c|}{ Stimulus Set } & \multicolumn{2}{|c|}{ Stimulus Set } & \multicolumn{2}{|c|}{ Stimulus Set } \\
\hline & Hue & Chroma & Hue & Value & Value & Chroma \\
\hline & + & - & + & - & + & - \\
\hline 1 & 32 & 23 & 14 & 44 & 14 & 45 \\
\hline 2 & 29 & 22 & 23 & 26 & 21 & 34 \\
\hline 3 & 13 & 44 & 21 & 29 & 13 & 48 \\
\hline 4 & 40 & 16 & 21 & 32 & 9 & 38 \\
\hline 5 & 30 & 23 & 34 & 18 & 9 & 44 \\
\hline 6 & 37 & 16 & 21 & 38 & 9 & 38 \\
\hline 7 & 27 & 30 & 8 & 47 & 21 & 31 \\
\hline 8 & 37 & 17 & 9 & 43 & 24 & 30 \\
\hline 9 & 37 & 23 & 14 & 28 & 48 & 16 \\
\hline 10 & 33 & 27 & 23 & 29 & 13 & 32 \\
\hline
\end{tabular}

Note-Entries represent the number of times a stimulus pair on the primary diagonal [e.g., $(1,6)$; see Figure 1$]$ was judged as more $(t)$ or less (-) dissimilar to the corresponding pair on the secondary diagonal [e.g., $(2,5)] . ~ N=72$ stimulus pairs. Unequal $N$ s are due to stimulus pairs that were judged as equally dissimilar. *Ten different subjects were assigned to each stimulus set.

The triads in which the salience of shared dimensional relations was varied provided a second assessment of perceptual organization and a more stringent measure of perceived dimensional relations. If the dimensional combinations of the hue-chroma, hue-value, and value-chroma stimulus sets could be analyzed into independent psychological dimensions in some or all of the triad types, using the classification task, the direction of our theoretical argument concerning the type of dimensional interaction evidenced by proximity data and the modifiability of perceptual analysis would change substantially. However, if components of color stimuli could not be analyzed into independent constituent dimensions in any of the triad types, then the perceived stimulus structure of color would appear to be one of consistent similarity organization and unanalyzability. That is, if subjects classified triads according to perceived overall similarity relations, regard- less of the dimension that provided the shared dimensional values and regardless of which color dimensions were combined, then we would conclude that subjects do not readily analyze color into component dimensions. The findings would allow a comparison with the results of Burns et al. (1978), which showed excellent agreement between scaling and restricted classification operations with separable and integral stimulus sets. By comparing such operations across the three sets of color stimuli, we could begin to address the generalizability of previously. reported convergence between scaling and classification and the issues surrounding the concept of degrees of analyzability (Burns, 1987; Burns et al., 1978; Garner, 1974; Lockhead, 1979; Smith \& Kemler, 1978).

\section{Method}

Subjects. A total of 36 Brown University undergraduates volunteered to participate in the classification studies. Approximately equal numbers of males and females were assigned to each stimulus set and each subject was paid $\$ 3.50$ for participation. Prior to the experimental session each subject was screened for color-vision deficiencies with the Ishihara test.

Stimuli. The three stimulus sets that can be generated by the combination of the three Munsell dimensions were used: hue-chroma, hue-value, and value-chroma. The $6 \times 6$ stimulus matrices described for each stimulus set in Experiment 1 were used.

Procedure. The subjecs were administered a version of the restricted classification task in which a triad of stimuli was presented on each trial and subjects were instructed to point to the two stimuli that "go best together." On half of the trials the stimuli were presented in a row; on the other half they were presented in a triangular array. The stimulus relations between adjacent stimuli were counterbalanced across trials. (See Shepp et al., 1980, for additional procedural details.)

The relations among the stimuli presented on a trial in this experiment are illustrated by the Type 1 and Type 3 triads in Figure 3 . In each of these triad types a classification based on shared dimensional relations is pitted against a classification based on overall similarity. The classification of stimuli A and B together indicates that a shared dimensional relation is perceived, whereas the classification of stimuli $\mathrm{B}$ and $\mathrm{C}$ together indicates the dominance of

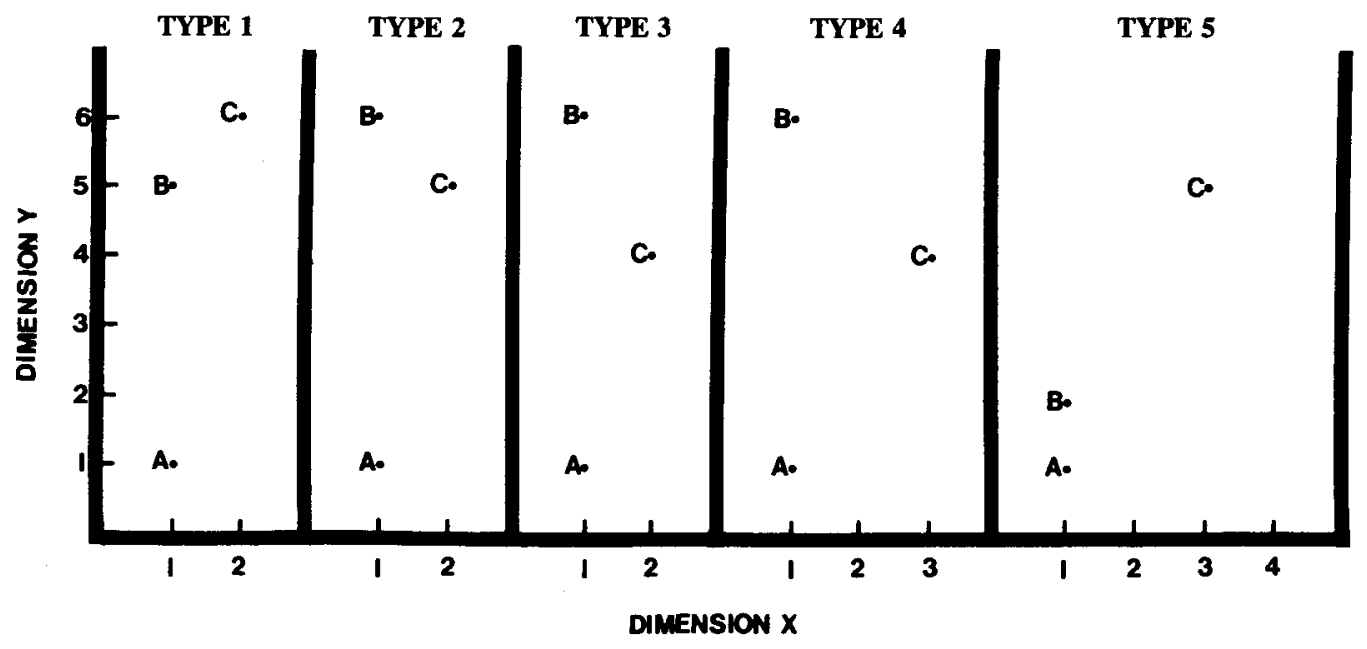

Figure 3. An illustration of the relations among stimuli on the five types of triads presented in Experiments 2-4. 
overall similarity relations. Subjects could also, of course, respond haphazardly by classifying $A$ and $C$ together.

In Type 1 triads, stimuli $A$ and $B$ share a level on dimension $X$ (see Figure 3) and are four levels apart on dimension Y. Stimuli $\mathrm{B}$ and $\mathrm{C}$ share no dimensional levels but are highly similar in that they differ only by one level on dimension $X$ and one level on dimension $Y$. The stimulus relations of Type 3 triads were arranged to increase the salience of perceived dimensional relations by decreasing the overall similarity classification. Stimuli $A$ and $B$ share a level on dimension $\mathrm{X}$ but are five levels apart on dimension $\mathrm{Y}$. Stimuli $B$ and $C$ are similar on both dimensions in that they are one level apart on dimension $\mathrm{X}$ and two levels apart on dimension $\mathrm{Y}$. If perceived dimensional relations and overall similarity relations in Type 1 triads are judged by a subject to be nearly equivalent in response to instructions to "put the two together that go best together," then the increase in dissimilarity between stimuli B and $C$ in Type 3 triads should decrease the similarity of the B $+C$ classification and lead to an increase in classifications based on shared dimensional relations. The effect of increased dimensional analysis in the context of decreased similarity of stimuli $\mathrm{B}$ and $\mathrm{C}$ has been supported in previous studies with adults (Burns et al., 1978), children (Shepp et al., 1980), and retardates (Burns, 1986).

Within each stimulus set, one of the dimensions presented a shared level on half of the trials, whereas the other dimension presented the overall similarity relation; the reverse arrangement was presented on the other half of the trials. Three unique Type 1 and Type 3 triads were constructed for each of the two dimensions composing the stimulus set. Each triad was presented eight times in a random order for a total of 96 trials.

\section{Results and Discussion}

Preliminary analyses revealed that there were no differences in classifications between stimuli presented in a linear array and those presented in a triangular array. Responses were averaged across these two types of displays and are summarized in Table 3, which shows the proportion of dimensional, haphazard, and overall similarity classifications for Type 1 and Type 3 triads in the hue-chroma, hue-value, and value-chroma stimulus sets. Two separate types of analyses were conducted on the data for each stimulus set. First, the proportion of overall similarity classifications made for each type of triad for shared values on each dimension of a stimulus set was compared to the level expected by chance (.33). Second, the numbers of overall similarity responses were compared factorially in a within-subjects analysis of variance where the factors were triad type ( 1 or 3 ) and shared dimensional level (e.g., hue or chroma).

The results from each type of analysis can be summarized easily. In all comparisons the proportions of overall similarity classifications exceeded chance levels [minimum correlated $t(11)=3.1, p<.02$ ]. The results of the analyses of variance showed no main effect due to type of triad or shared dimension for any of the stimulus sets ( $p s>.05$ ). Moreover, there were no significant interactions between these two factors in any of the analyses ( $p s>.05$ ).

The results of the present experiment show that the dominant basis of classification for the three stimulus sets, hue-chroma, hue-value, and value-chroma, was overall similarity. Although it appears that there was some ten- dency for subjects to classify less by overall similarity in Type 3 than in Type 1 triads (see Table 3), the effect was not reliable. It is important to note that the tendency to ignore shared dimensional relations and to classify by overall similarity relations contrasts sharply with the results of Burns et al. (1978), Handel and Imai (1972), and others for stimulus sets that have a perceived dimensional structure according to other criteria. For such sets of separable stimuli (e.g., circle size and angle of radial line, size and brightness) the dominant form of classification is according to shared dimensional relations.

The results of Experiment 2, using the three combinations of the dimensions of color, indicate that the stimuli are perceived holistically; the results converge with the results and interpretation of Experiment 1 that stress the lack of independent dimensional structure composing these stimuli. However, the results from the restricted classification task do not appear to reflect the differential types of dimensional analysis suggested by the scaling procedures. In contrast to Experiment 1 , in which valuechroma and hue-value judgments showed a mutual interaction whereas hue-chroma judgments revealed an asymmetric interaction, the present findings using the classification task reflect no differential types or differential degrees of dimensional analysis for the three combinations of color dimensions.

\section{EXPERIMENT 3}

The results of Experiments 1 and 2 showed that the stimuli generated by combining the dimensions of the Munsell color system were not perceived as conjunctions of features on subjectively independent dimensions; instead, such stimuli were perceived as integral wholes. Obviously, holistic perceptual organization was the initial and dominant mode of organization, but we could not rule

Table 3

Proportions of Dimensional (D), Haphazard (H), and Similarity (S) Responses in the Restricted Classification Task for Each Dimension Composing the Hue-Chroma, Hue-Value, and Value-Chroma Stimulus Sets

\begin{tabular}{|c|c|c|c|c|c|c|}
\hline Triad Type & D & $\mathbf{H}$ & $S$ & D & H & $S$ \\
\hline & \multicolumn{6}{|c|}{ Hue-Chroma Set } \\
\hline & \multicolumn{3}{|c|}{ Shared Hue } & \multicolumn{3}{|c|}{ Shared Chroma } \\
\hline 1 & .06 & .03 & .91 & .04 & .02 & .94 \\
\hline \multirow[t]{3}{*}{3} & .08 & .06 & .86 & .07 & .09 & .84 \\
\hline & \multicolumn{6}{|c|}{ Hue-Value Set } \\
\hline & \multicolumn{3}{|c|}{ Shared Hue } & \multicolumn{3}{|c|}{ Shared Value } \\
\hline 1 & .14 & .08 & .78 & .04 & .06 & .90 \\
\hline \multirow[t]{3}{*}{3} & .24 & .13 & .63 & .14 & .05 & .81 \\
\hline & \multicolumn{6}{|c|}{ Value-Chroma Set } \\
\hline & \multicolumn{3}{|c|}{ Shared Value } & \multicolumn{3}{|c|}{ Shared Chroma } \\
\hline 1 & .09 & .08 & .83 & .10 & .17 & .73 \\
\hline 3 & .10 & .10 & .80 & .23 & .15 & .62 \\
\hline
\end{tabular}


out the possibility that there might be circumstances under which dimensional structure would emerge as the basis for perceptual organization.

In Experiment 3, we attempted to identify some of these circumstances. It was clear from previous research that the restricted classification task provided the most unambiguous evidence to support the claim that stimuli are perceived according to dimensional relations. When stimuli were perceived as conjunctions of features, subjects showed a strong preference to classify together those stimuli that shared an identical feature. In the present experiment, two operations were added to the restricted classification task described in Experiment 2 to promote the dimensional analysis of color stimuli: (1) instructions and training on dimensional analysis, and (2) additional triad types that served to increase the salience of the shared dimensional relations. The subjects were first given instructions concerning the concept of a shared dimensional relation and were trained and tested on dimensional analysis, using stimuli that varied in height and width. Then they were given new training and feedback on the color dimensions that were varied in the classification task. Both the training and the experimental triads varied the salience of dimensional relations beyond the variations attempted in Experiment 2.

\section{Method}

Subjects. For each stimulus set, the subjects were 12 Brown University undergraduates ( 6 male, 6 female) who volunteered to participate; they were paid $\$ 3.50$ for the session. The subjects were pretested for color-vision deficiencies with the Ishihara Test.

Stimuli. The three stimulus sets of Munsell colors that had been used in Experiment 2 were also used in Experiment 3. The dimensional-analysis training stimuli consisted of a $6 \times 6$ matrix of objects varying in height $(2.1,2.5,3.0,3.6,4.3$, and $5.2 \mathrm{~cm})$ and width $(6.2,7.5,9.0,10.8,13.0$, and $15.6 \mathrm{~cm})$.

Procedure. Before training and testing on the attributes of color was begun, an analogous training and testing session was completed using the dimensions of height and width of rectangle. The dimensions of height and width were described verbally and examples of stimuli illustrating shared height and shared width relations were shown to the subject. Once the task of classifying by shared height or width was well understood, a series of experimental trials were presented which included Type 2, Type 4, and Type 5 triads (described below). These procedures were included to further underscore the task demands of dimensional analysis and to obtain a measure of the effectiveness of our procedures with a highly analyzable stimulus set (see Shepp et al., 1980).

The subjects were given an extensive description of the dimensions of color. As an example, the instructions for the hue-brightness set are given below. Subjects assigned to the classification of the other stimulus sets received analogous instructions.

This is an experiment involving two aspects of color: hue and brightness. Hue refers to the color's wavelength or what we often think of as the name of a color. Hue can range from reds to yellows to blue, etc. [At this point an illustration of a horizontal section of the color sphere at the equator was shown (from Itten, 1970, p. 68).] Brightness refers to the amount of light reflected from the hue. If brightness were to be arranged on a scale from black to white, there would be equal steps of gray from dark gray to almost white [The experimenter illustrated this point by showing a vertical section of the color sphere from white to black.] By looking at this color sphere (Itten, 1970, p. 69), we immediately notice two things. If we look at the gray center square and move one step to the right and one step to the left we see that these represent two different hues that share the same level of brightness. The same is true for any horizontal level of the sphere. Along any vertical line in the sphere the stimuli share a level on hue and differ in brightness. [The subjects were given time to study the color sphere.] Now we will examine a set of actual Munsell chips that vary in hue and brightness.

[Subjects were presented with a set of Munsell chips that varied in six levels of hue in the blue range and six levels of value (brightness) in a $6 \times 6$ matrix. Chips were pointed out that shared a level on each dimension. The matrix of chips was removed. A series of Type 2 and Type 4 triads were then presented and subjects were asked to classify by a shared level on hue or brightness. Classifications were corrected and additional triads were presented until the subjects appeared to understand the task demands as well as the concept of psychologically independent dimensions.]

Now we are ready to begin the experiment on classification by the dimensions of hue and brightness. On each trial you will be presented with three color chips. Two out of each triad of chips will share the same level on either hue or brightness. Your task is to choose the two stimuli that share a level on one of these two dimensions. There are no tricks, although some trials may be harder than others. There will be a total of 72 trials for you to classify in this way.

The relations between the stimuli in Type 2 and Type 4 triads (see Figure 3) pit dimensional against overall similarity classifications. In this experiment, the Type 2 and Type 4 triads were of two types: extended and nonextended. In both of these arrangements stimuli $A$ and $B$ share an identical value on one dimension and differ considerably on the second. The difference between stimuli $A$ and $B$ in extended triads is five steps, whereas the difference in nonextended triads is three steps.

The structure of the Type 2 triad is similar to that of the Type 1 triad that was used in Experiment 2. The change in the B-C stimulus relations from the primary to the secondary diagonal was required to accommodate the extended-nonextended manipulation. The relations between the stimuli of Type 4 triads were arranged in such a way as to increase the salience of dimensional relations within the triad: the salience of dimensional relations was varied by changing the similarity relations between stimulus $C$ and stimuli $A$ and $B$. When stimuli A and B were extended, stimulus $C$ differed from $B$ by two levels on each dimension. When stimuli $A$ and $B$ were nonextended, stimulus $\mathbf{C}$ differed from stimulus $B$ by two levels on dimension $\mathrm{X}$ and one level on dimension $\mathrm{Y}$.

The presentation of Type 5 triads permitted assessment of the degree to which dimensional classifications for Type 2 and Type 4 triads were due to a particular strategy not based on perceptual factors. The illustration and training triads had been of Types 2 and 4. In those phases of the experiment, subjects might have observed that often the stimuli that shared a dimensional feature (i.e., A and B) were also the stimuli that were most dissimilar. Subjects who made this observation might have chosen to classify stimuli $A$ and $B$ together in response to the instruction to make dimensional classifications, even though no dimensional relations were actually perceived. In Type 5 triads, stimuli A and B shared an identical value on one dimension and differed by only one level on the second; stimulus $\mathrm{C}$ differed from stimulus $\mathrm{B}$ by three levels on one dimension and two levels on the other. If the subjects could recognize a shared dimensional relation, $\mathrm{A}$ and $\mathrm{B}$ should continue to be classified together. If, on the other hand, the subjects were attempting to classify on the basis of the rule of maximal dissimilarity, then stimulus $\mathrm{C}$ should be classified with either A or B.

For Type 2 and Type 4 triads, there were six extended and six nonextended triads for each dimension in each color set. For Type 5 triads, there were six different triads for each dimension and each was repeated once during the series of trials. Each subject classi- 
fied a total of 72 triads that were presented according to either of two random sequences. In all other ways, the procedure followed that of Experiment 2.

\section{Results and Discussion}

One of the first questions to answer in this experiment was whether subjects have difficulty in understanding the general concepts of shared dimensional relations, and whether they can readily apply these concepts when instructed to do so in a restricted classification task. In the training phase of this experiment, when instructed to classify rectangles in terms of shared dimensional relations, the subjects were able to classify accurately stimuli that shared either height or width. The percentages of dimensional classifications for Type 2, Type 4 , and Type 5 triads were in excess of $99 \%$. These results indicate, first, that rectangle stimuli can be decomposed into the dimensions of height and width even though the primary response is based on overall similarity relations (e.g., Shepp et al., 1980), and second, that our subjects had a clear understanding of the task demands of instructed classification.

The results of the instructed classification task using color stimuli are summarized in Table 4 . A preliminary analysis indicated that the classifications of extended and nonextended triads showed no significant differences ( $p s>.05$ ). Thus, the data for the two types of triads were combined and the overall proportions for each type of classification are presented. Our hypothesis was that instructions to classify the triads by shared dimensional relations should promote the dimensional analysis of color stimuli. In fact, if color stimuli could be analyzed into component dimensions, there should be a significant proportion of classifications based on shared dimensional relations in this experiment.

An inspection of Table 4 shows that instructed classification of color stimuli does not yield the same pattern of results as instructed classification of rectangles. For Type 2 triads, the instructions to classify by a shared dimensional relation resulted in fewer classifications by shared overall similarity than in Experiment 2, but $t$-test comparisons indicated that the proportions of overall similarity classifications were significantly greater than chance levels (.33) in five of six comparisons (all $t \mathrm{~s}>$ 3.2 , ps <.01). The exception was noted in the value-chroma set. When the subjects were given the opportunity to classify triads that had a shared level on the dimension of value, they produced a distribution of classifications that did not differ from chance $(p>.05)$. Overall, these results indicate that the instructions to classify by shared dimensional relations did not increase the proportion of dimensional classifications to greater than chance levels on Type 2 triads.

Type 4 triads were designed to promote dimensional analysis by increasing the dissimilarity of the objects for the similarity $(B+C)$ classification. An inspection of the proportions of classifications of Type 4 triads indicates that the instructions to classify by shared dimensional re-
Table 4

Proportion of Dimensional (D), Haphazard (H), and Similarity (S) Responses in the Restricted Classification Task (Instructed Condition) for Each Dimension Composing the Hue-Chroma, Hue-Value, and Value-Chroma Stimulus Sets

\begin{tabular}{|c|c|c|c|c|c|c|}
\hline Triad Type & D & $\mathbf{H}$ & $\mathbf{S}$ & $\mathrm{D}$ & $\mathbf{H}$ & $\mathbf{S}$ \\
\hline & \multicolumn{6}{|c|}{ Hue-Chroma Set } \\
\hline & \multicolumn{3}{|c|}{ Shared Hue } & \multicolumn{3}{|c|}{ Shared Chroma } \\
\hline 2 & .01 & .35 & .64 & .30 & .23 & .47 \\
\hline 4 & .10 & .42 & .48 & .59 & .15 & .26 \\
\hline 5 & .63 & .06 & .31 & .74 & .05 & .21 \\
\hline \multirow[t]{3}{*}{$5^{\prime}$} & .95 & .02 & .03 & .92 & .04 & .04 \\
\hline & \multicolumn{6}{|c|}{ Hue-Value Set } \\
\hline & \multicolumn{3}{|c|}{ Shared Hue } & \multicolumn{3}{|c|}{ Shared Value } \\
\hline 2 & .07 & .22 & .71 & .33 & .14 & .53 \\
\hline 4 & .30 & .21 & .48 & .63 & .09 & .28 \\
\hline 5 & .81 & .06 & .13 & .71 & .02 & .27 \\
\hline \multirow[t]{3}{*}{$5^{\prime}$} & .88 & .09 & .03 & .92 & .05 & .03 \\
\hline & \multicolumn{6}{|c|}{ Value-Chroma Set } \\
\hline & \multicolumn{3}{|c|}{ Shared Value } & \multicolumn{3}{|c|}{ Shared Chroma } \\
\hline 2 & 31 & .27 & .42 & .16 & .28 & .56 \\
\hline 4 & .40 & .25 & .35 & .36 & .28 & .36 \\
\hline 5 & .59 & .17 & .34 & .66 & .15 & .23 \\
\hline $5^{\prime}$ & .96 & .00 & .04 & .93 & .04 & .03 \\
\hline
\end{tabular}

Note-Type $5^{\prime}$ indicates classifications of Type 5 triads by subjects not instructed in dimensional analysis.

lations differentially affected the proportion of dimensional classifications for the three stimulus sets. In classifying triads from the value-chroma set, the subjects did not classify consistently ( $p$ s $>.05$ ) by any of the three types of stimulus relations (dimensional, haphazard, or similarity). For Type 4 triads, it appears that shared value or chroma relations cannot be extracted under our dimensional analysis conditions.

In contrast, the classifications of Type 4 triads in the hue-chroma and hue-value sets show an asymmetric effect. In both sets, when subjects were presented with stimuli that shared a common hue, the proportion of overall similarity responses still exceeded chance levels ( $t \mathrm{~s}>2.2, p \mathrm{~s}<.05)$. When the stimuli shared either a common chroma or value, however, the subjects showed a significant tendency to classify by shared dimensional relations $(t \mathrm{~s}>4.0, p s<.01)$. The results for Type 4 triads reveal that shared chroma and shared value can be extracted and employed as the basis for classification when combined with hue.

As shown in Table 4, the proportions of dimensional, haphazard, and similarity classifications for Type 5 triads reveal a pattern that does not support the idea that the subjects used a strategy of classifying the most dissimilar objects together. Although such a strategy seemed unlikely, it was important to rule out the possibility that this was the basis for increased dimensional classifications on instructed classification triads.

A second type of analysis was employed in order to compare the classifications of Type 5 triads by subjects who were instructed to classify by shared dimensional re- 
lations with classifications that occurred without instruction. An independent group of $\mathbf{8}$ subjects was tested using the procedures described in Experiment 2. These subjects classified Type 5 triads (mixed with Types 2 and 4) under instructions to "classify together the two stimuli that appear to go together best." The classifications of Type 5 triads by the "uninstructed"' subjects (i.e., subjects not instructed in dimensional analysis) are presented in Table 4 as Type $5^{\prime}$. In all cases, these subjects classified reliably according to shared dimensional relations ( $t \mathrm{~s}>12.0, p \mathrm{~s}<.001$ ), a result that is not surprising, considering that the stimuli that shared dimensional levels were also the most similar overall. In the instructed classification condition, the proportion of dimensional classifications of Type 5 triads was significantly less than the proportion exhibited by the uninstructed subjects in all cases but one (all $p s<.001$ ). The exception occurred for the hue-value set when the subjects classified triads sharing a common hue; in this instance, there was no difference between the proportions of dimensional classifications made by instructed and uninstructed subjects. Generally, the differences in the proportions of dimensional classifications made by the two groups of subjects indicate that the subjects instructed to classify by shared dimensional relations were not simply classifying in terms of overall similarity. They appeared to be attempting to analyze the objects, without much success.

To summarize, the results of Experiment 3 are quite clear. When the subjects were instructed to classify by dimensional relations, overall similarity relations continued to dominate classifications on Type 2 triads. The proportions of such classifications were, however, significantly reduced in comparison with analogous triads (Type 1) in Experiment 2. In contrast, on the Type 4 triads, the instructions and training increased the salience of dimensional relations for some attributes of color. When stimuli varied in value versus chroma, subjects continued to be unable to identify stimuli that shared a common dimensional level on value or chroma. However, when either value or chroma was varied with hue, the stimuli that shared a level on chroma or value were identified on a significant proportion of trials. Yet there was an unquestionable asymmetry; in neither set could the subjects identify stimuli that shared a common level on hue.

Our results using restricted classification procedures provide more detailed information about the organization and representation of color stimuli. The instructed classification task was sensitive enough to pick up differences in the degree and type of dimensional interaction. A comparison of scaling and classification results for these three stimulus sets produces a more complicated picture. The patterns of instructed classifications for hue-value and hue-chroma were very similar and differed greatly from the pattern exhibited by subjects instructed to analyze value-chroma stimuli. Scaling procedures identified a different type of dimensional interaction for hue-chroma than for hue-value and value-chroma. For the hue-chroma set, judgments of chroma appeared to be un- affected by changing shared levels of hue. This supports the idea that chroma may be extracted and represented as an independent dimension when combined with hue. The subjects' ability to extract shared chroma and not shared hue relations was also mirrored in the instructed classification data. Similarly, the mutual interaction evidenced by the dissimilarity judgments of value-chroma stimuli was supported by the subjects' inability to extract shared dimensional relations in classification. The hue-value data from scaling and classification do not converge so readily. The scaling analysis reflects mutual interaction; the classification task reflects subjects' ability to extract shared value relations only. Shared hue relations could not be identified on these triads. Additional studies are needed to identify the implications of these different profiles of dimensional interactions for operations of dimensional analysis. In Experiment 4, we extended the question of the analysis of color stimuli a step further. Experiment 3 showed that instructions to classify by dimensions did not promote analysis. Our question in Experiment 4 was whether such instructions given to individuals who had been tutored in hue, saturation, and color ("color experts") would lead to the increased analysis of color.

\section{EXPERIMENT 4}

The subjects of Experiments 1-3 were undergraduates with no special training or experience in analyzing color attributes beyond those opportunities provided by the experiment. It is, of course, plausible that special aptitude and/or extended training influences the ability to analyze color stimuli into their component features. In Experiment 4 , this hypothesis was tested with a group of studentartists enrolled in the Rhode Island School of Design and one color-vision scientist from Brown University. The students were enrolled in a color theory course that devoted considerable time to the general analysis of color attributes, and to the Munsell color series specifically. At the conclusion of the course, the students participated in our experiment, which employed instructed classification procedures with the hue-chroma stimulus set.

\section{Method}

Subjects. The subjects were 11 students ( 6 female, 5 male) from the Rhode Island School of Design who volunteered to participate and were paid $\$ 3.50$ for the session. In addition, one color-vision scientist from Brown University agreed to participate. All subjects were pretested with the Ishihara Test to reveal any color-vision deficiencies.

Stimuli. The Munsell hue-chroma stimulus set that was employed in Experiment 3 was used. In addition, a training task employed a $6 \times 6$ set of squares varying in size $(1.9,2.4,3.0,3.9,4.9$, and $6.2 \mathrm{~cm}$ ) and value (brightness) $(3,4,5,6,7$, and 8). Dissimilarity relations of the latter stimuli previously reported by Burns et al. (1978).

Procedure. The procedures employed in Experiment 3 were repeated in Experiment 4. Before being trained on dimensional analysis with color stimuli, the subjects were trained and tested on stimuli varying in size and brightness. These stimuli, rather than stimuli 
varying in height and width, were employed in order to extend the examination of the effectiveness of dimensional analysis training. Measures of spontaneous classification of size versus brightness with adult subjects had previously been reported (Burns et al., 1978), so that a measure of the effectiveness of our training in the present experiment could be obtained. As in Experiment 3, a set of color stimuli varying in alternative hue and chroma levels was used in the training and feedback procedures for the color attributes of hue and chroma.

\section{Results and Discussion}

The training and instructions to classify by shared dimensional relations using the size-brightness set yielded dimensional classifications for Type 2, Type 4, and Type 5 triads that approached $100 \%$. Uninstructed classification of the same stimuli previously yielded approximately 82\% dimensional responding (Burns et al., 1978). That the present instructions produced significant increases in the dimensional analysis of even separable stimuli is noteworthy and supports our assumption that such instructions were well understood.

The proportions of dimensional, haphazard, and overall similarity classifications for the hue-chroma stimulus set are shown in Table 5. For Type 2 triads, the instructions to classify by a shared dimensional level produced a pattern of chance-level responding for triads that presented a shared level on chroma $(p>.05)$, and reliable overall similarity classifications for triads that presented a shared level on hue $[t(11)=3.02, p<.01]$. The color experts classified by shared dimensional relations on $10 \%$ of the shared-hue triads and $42 \%$ of the shared-chroma triads. Type 4 triads revealed a different pattern; triads presenting a shared level on hue yielded a distribution that does not differ from chance $(p>.05)$, whereas the classifications of triads that presented a shared level on chroma yielded a significant proportion of dimensional classifications $[t(11)=4.3, p<.01]$. The asymmetric pattern of dimensional analysis was evident in these data (38\%, hue; $76 \%$, chroma), just as in those for Experiment 3.

Type 5 triads showed a reliable pattern of classification by shared dimensional levels for triads that presented stimuli that shared either hue or chroma $(t \mathrm{~s}>3.2$, ps $<.01)$. As in Experiment 3, the possibility that the subjects had classified stimuli on the basis of a leastdissimilar rule was not supported by the data from these triads.

General comparisons between the color experts (Experiment 4) and nonexperts (Experiment 3) suggest that

Table 5

Proportion of Dimensional (D), Haphazard (H), and Similarity (S) Responses by Color Experts for Each Dimension Composing the Hue-Chroma Stimulus Set in the Instructed Classification Task (Experiment 4)

\begin{tabular}{cccccccc} 
& \multicolumn{3}{c}{ Shared Hue } & & \multicolumn{3}{c}{ Shared Chroma } \\
\cline { 2 - 4 } \cline { 5 - 7 } Triad Type & D & H & S & & D & H & S \\
\hline Type 2 & .10 & .42 & .48 & & .42 & .28 & .30 \\
Type 4 & .38 & .30 & .32 & & .76 & .08 & .16 \\
Type 5 & .67 & .07 & .26 & & .69 & .04 & .27 \\
\hline
\end{tabular}

Table 6

Proportion of Dimensional (D), Haphazard (H), and Similarity (S) Responses by Color Experts (Experiment 4) and Nonexperts (Experiment 3) for Type 4 Triads That Presented a Shared Chroma Relation at Low and Medium-to-High Levels of Chroma

\begin{tabular}{lccccccc}
\hline & \multicolumn{3}{c}{ Color Experts } & & \multicolumn{3}{c}{ Nonexperts } \\
\cline { 2 - 6 } \cline { 5 - 8 } Degree of Chroma & D & H & S & & D & H & S \\
\hline Medium to High & .91 & .00 & .09 & & 62 & .10 & .28 \\
Low & .48 & .27 & .25 & & .52 & .23 & .25 \\
\hline
\end{tabular}

neither special aptitude nor special experience played an important role in the analysis of the attributes of color. Both groups of subjects successfully analyzed stimuli that shared a common chroma; neither group successfully analyzed stimuli that shared a common hue. One may argue, however, that color experts showed a trend toward analyzing chroma on Type 2 triads, since the experts' pattern of classifications did not differ from chance, whereas the majority of classifications by nonexperts were ones of overall similarity $(p<.05)$. Similarly, Type 4 triads in which a shared level on hue was present yielded a predominance of similarity classifications by nonexperts and a pattern that did not differ from chance for experts. In both cases the difference between nonexperts and experts was in the direction compatible with our hypothesis.

More fine-grained analyses of our data suggest some additional differences between the performance of color experts and that of nonexperts. Table 6 shows the classifications of both groups for Type 4 triads that included stimuli with a common chroma, shown separately for triads composed of stimuli with very low chroma levels (chromas 2 and 4) and for those with medium-to-high chroma levels (chromas 6-12). When stimuli are generated by combining levels of chroma from the low end of the scale, the subject's task is to identify a common chroma in a set of achromatic stimuli (i.e., grayish). As the chroma level increases, the stimuli become increasingly chromatic. As shown in Table 6, both color experts and nonexperts were able to classify by shared dimensional relations regardless of the degree of chroma presented in the stimuli (all $t \mathrm{~s}>3.01$, ps $<.01$ ); however, dimensional relations were more easily perceived in the triads composed of stimuli with mediumto-high levels of chroma. Moreover, at the medium-tohigh levels of chroma, experts detected dimensional relations better than did nonexperts. An analysis of variance for a mixed factorial design comparing the percentages of dimensional responses at two levels of chroma (low and medium-to-high) and two levels of color expertise revealed a main effect of degree of chroma $[F(1,22)$ $=23.98, p<.001]$ and an interaction of expertise and degree of chroma $[F(1,22)=8.21, p<.01]$.

The fact that the color experts did better than the nonexperts in recognizing chroma relations among the stimuli is not particularly surprising and may be due to both skill and experience. Differences between the two groups could certainly arise due to individual differences in talent. Students who are admitted to the Rhode Island School of Design are selected, among other reasons, for their proven 
artistic talent. Such talent could easily be correlated with visual sensitivity to color. It is also likely, however, that perceptual experience of the sort described by Gibson (1969) could contribute to the difference. Although both groups of subjects had a strong operational command of the concepts of color dimensions, the color experts had substantially more experience in analyzing aspects of color

\section{GENERAL DISCUSSION}

On the basis of our analysis of two types of judgments of similarity (i.e., scaling and restricted classification procedures), it is clear that current thinking concerning the perceptual organization of color attributes should be further revised. The general idea that color experiences are not organized in terms of the independent psychological dimensions of hue, brightness, and saturation has already been well established. Shepard (1964) described color chips as unitary stimuli, which are processed as homogeneous wholes, compared with analyzable stimuli, which are analyzed into component dimensions. Garner $(1970,1974)$ further formalized and extended the notions of unitary and analyzable stimuli in his distinction between integral and separable stimuli. The present findings suggest a picture of the perceptual organization of color that is a further refinement of the concepts of integrality and separability. We have shown that particular attributes of color stimuli combine with each other to produce varying patterns of interaction in conscious perceptual experience. These forms of dimensional interaction are mirrored in the patterns of scaling data as well as in the instructed restricted classification data.

Before discussing these findings in detail, it is necessary to clarify some general issues regarding our selection of methodology and analysis. As stated above, the distinction between integral, or unitary, stimuli and separable, or analyzable, stimuli has had a long history. One of the distinctions between these two stimulus types is based on spatial metrics. The adherence of scaling data to a Euclidean metric has been indicative of integral stimuli, whereas the adherence to a city-block metric has been indicative of separable stimuli. As Foard and Kemler Nelson (1984) pointed out, the distinction between Euclidean and city-block metrics has been associated with the fundamental criterion of privileged axes initially described by Garner (1974) and Torgerson (1958). We wish to focus here on the pattern of findings for spatial metrics and integral stimuli. Color stimuli, often described as the best example of integral stimuli, have been shown by Torgerson (1958), Shepard (1964), Hyman and Well $(1967,1968)$, and others to yield dissimilarity judgments based on a Euclidean metric. Dunn (1983) recently reviewed the scaling literature and concluded that, in fact, the only dimensions that yield dissimilarity in terms of a Euclidean metric are hue, saturation, and brightness. In sum, the idea that the geometry of psychological space (i.e., spatial metrics) can predict psychological integrality is well entrenched in the literature.

\section{Assumptions of Psychological Space}

There is a serious problem with the use of spatial metrics as a converging operation for the characterization of stimulus structure. A number of assumptions about psychological space must be met before an analysis of the geometry or metric of that psychological space is appropriate. One of the most basic assumptions, that of interdimensional additivity, has been shown in a number of studies to be violated when the dimensions composing objects are integral as defined by other types of tasks (Burns, 1976; Burns et al., 1978; Krantz \& Tversky, 1975; Wender, 1971; Wiener-Ehrlich, 1978). If the interdimensional additivity assumption is violated and two dimensions interact in judgments of similarity, the application of combination rules or metrics for two independent dimensions is not meaningful. Given this is true, we need to reconsider the previous work that established this postulate that similarity judged according to a Euclidean metric is diagnostic of integral stimuli. Clearly, if the underlying assumptions of psychological space are violated, the adherence to a metric as part of an operational definition for stimulus integrality is unfounded.

There are additional problems with the use of spatial metrics and concepts of integrality. Several investigators have shown that even when these assumptions about psychological space are met, detailed analyses of individual spatial metrics do not distinguish between integral and separable stimuli. Dunn (1983) showed that even similarity judgments of prototypical separable stimuli (i.e., circle size and angle of radial line) that $d o$ meet the assumptions of interdimensional additivity (see also Burns et al., 1978) do not satisfactorily conform to a city-block or Euclidean metric. Ronacher and Bautz (1985) also showed that particular metrics should not be associated with particular types of stimulus structure. In detailed analyses of 27 subjects who judged dissimilarity of size-brightness stimulus pairs, Ronacher and Bautz found that data from 13 subjects fit the city-block metric, data from 11 subjects fit the Euclidean metric, and data from 3 subjects fit an intermediate metric. Eighty percent of these subjects produced data that supported the assumptions of interdimensional additivity. Clearly, the relationship between acceptance of interdimensional additivity and spatial metrics is not simple; acceptance does not imply the use of a particular metric, but is simply a necessary condition for the applicatior of a metric.

There is enough evidence to alter current thinking about the use of spatial metrics for studies of stimulus integrality. Beginning with the work of Burns (1976; Burns et al., 1978), the emerging pattern regarding concepts of stimulus structure and scaling procedures is one in which the tests based on the ADMM can provide an alternative diagnostic to spatial metrics for concepts of integrality and separability. Since the tests of the ADMM are independent from tests of spatial metrics, the use of metrics may be considered as a second step for dimensions that meet such tests. It is proposed that similarity judgments of integral stimuli violate the assumptions of dimensional independence, in contrast to separable stimuli, which meet 
these assumptions. On the basis of the present findings with three color sets, we can add that the form of violation (e.g., symmetric vs. asymmetric interaction) can provide additional information concerning internal representation.

\section{The Present Findings}

In Experiment 1, analyses of the patterns of dissimilarity judgments of each dimensional set reflected particular types of interactions, as described by the ADMM. Data from the hue-chroma set yielded an asymmetric interaction, in contrast to the mutual interaction shown by the hue-value and value-chroma sets. The two-dimensional scaling configurations for each stimulus set, although appropriate according to Munsell notation, do not reflect these interactions and should not be interpreted as support for dimensional structure.

The results of restricted classification in Experiment 2 support the claim that color stimuli are primarily perceived as unitary integral wholes, and not as conjunctions of hue, chroma, and value dimensions. Given triads of stimuli such as those shown as Type 1 and Type 2 in Figure 3, the subjects classified stimuli $B$ and $C$ together far more than they classified A and B together. The former type of classification is based on similarity on each dimension, whereas the latter is based on identity on one dimension and considerable dissimilarity on the other dimension. Classification by overall similarity is typical of interacting dimensions, in contrast to classification by a shared dimensional component, which is characteristic of perceptually separate dimensions (e.g., Burns et al., 1978; Garner, 1974; Handel \& Imai, 1972).

In the data of Experiments 3 and 4 the profile of integrality and perceptual modifiability for color stimuli becomes clearer. These data indicate that subjects with additional training and considerable skill and talent cannot perceptually analyze color stimuli in such a way that shared levels on hue can be identified, but they can access shared chroma or shared value levels in the context of variations of hue. Hue-chroma dimensional combinations produce stimuli that can be analyzed in such a way that shared chroma relations, especially at moderate or high saturation levels, can be detected in the classification task. In sharp contrast, the value-chroma dimensions combine to produce stimuli that cannot be analyzed in the classification task. Hue-value stimuli fall between these two extremes; shared value can be identified in classification to a moderate degree (not as successfully as shared chroma in the hue-chroma set).

\section{Converging Operations}

We believe that our findings in the present study reveal new information about the convergence and sensitivity of dissimilarity judgment and classification tasks and suggest a divergence of our tasks from Garner's (1974) converging operations that operationally define integral (and separable) stimulus structure. Both of our tasks focus on the ability to consciously analyze perceptual ex- perience. As Smith and Baron (1981) pointed out, tasks that focus on similarity relations (e.g., scaling and classification) reflect consistent measures of a subject's tendency to respond on the basis of dimensional identity, and not of the subject's ability to do so. In Experiments 1 and 2 , we tapped adults' preference for processing information about the dimensions of color stimuli. In Experiments 3 and 4, we altered task requirements and focused on the subjects' ability to extract shared dimensional identities. The pattern of performance in the instructed classification task, however, reflected aspects of the original scaling data. This finding does not support the notion that qualitatively different mechanisms are responsible for adults' preference for processing information (or cognitive/perceptual style) and adults' ability to process such information. Additional investigations are needed that focus on the relationship between similarity judgments and various classification procedures (both speeded and unspeeded) in order to better understand the process by which objects are perceived and/or classified as similar to or different from one another. We share the concern of Smith and Baron (1981) and Dunn (1983) that current models of processing that distinguish between integrality and separability on the basis of converging operations are too general and do not address issues concerning the commonality or distinctiveness of such mechanisms or successfully account for experiences that influence the development of such mechanisms.

Our results may provide an alternative account for some recent findings by Foard and Kemler Nelson (1984), who also focused on the dimensional analysis of color. Foard and Kemler Nelson's subjects were able to identify whether pairs of stimuli varied on one dimension (e.g., value1-chroma2 and value4-chroma2) or on two dimensions (e.g., value1-chroma2 and value4-chroma4) of color. Subjects rated the complexity of the two stimuli (i.e., whether the stimuli varied on one dimension or two), and overall similarity between the two sets of stimuli was controlled to avoid the confound between complexity and dissimilarity. The subjects were moderately successful in recognizing stimulus pairs that were varied on one dimension as compared with two dimensions. We are concerned about whether such findings reflect subjects' abilities to analyze dimensions of color into psychologically independent components. It seems that a true characterization of the relationship between the ability to quantify dimensional differences and the ability to identify shared dimensional components will require studies that vary hue-value and hue-chroma dimensions in addition to value-chroma dimensions, as well as task demands.

The present results suggest that integral stimuli vary in types of dimensional interactions. The differing types of interaction are reflected in our subjects' ability to identify identical levels on dimensions composing color stimuli, as well as in their judgments of dissimilarity. We suggest a new diagnostic for stimulus integrality, based on the assumptions of the ADMM (Beals et al., 1968; Krantz \& Tversky, 1975; Tversky \& Krantz, 1969, 1970). 
This new diagnostic, which can specify types of interactions, represents a new level of refinement for characterizing perceptual organization and goes beyond the previous concept of integrality. Further work is needed to characterize the relations among the various factors explored in the present study (i.e., stimulus characteristics, task demands, subject factors) and the common and distinctive mechanisms underlying such factors.

\section{REFERENCES}

Beals, R., Krantz, D. H., \& Tversky, A. (1968). Foundations of multidimensional scaling. Psychological Review, 75, 127-142.

Boynton, R. M., \& GoRDON, J. (1965). Bezold-Brücke hue shift measured by color-naming technique. Journal of Optical Society of America, 55, 78-86.

BURNS, B. (1976). Distinctions between proximity data from integral and separable dimensions on the basis of the formal properties of the additive-difference model. Unpublished manuscript, Brown University, Providence, RI

BURNS, B. (1986). Relationship of perceived stimulus structure and intelligence: Further tests of a separability hypothesis. American Journal of Mental Deficiency, 91, 196-200.

BurNs, B. (1987). Is stimulus structure in the mind's eye? An examination of dimensional structure in iconic memory. Quarterly Journal of Experimental Psychology, 39A, 385-408.

Burns, B., Shepp, B. E., McDonough, D., \& Wiener-Ehrlich, W. (1978). The relation between stimulus analyzability and perceived dimensional structure. In C. H. Bower (Ed.), The psychology of leaming and motivation: Advances in research and theory (Vol. 12). New York: Academic Press.

DUNN, J. C. (1983). Spatial metrics of integral and separable dimensions. Journal of Experimental Psychology: Human Perception \& Performance, 9, 242-257.

Foard, C. F., \& Kemler Nelson, D. G. (1984). Holistic and analytic modes of processing: The multiple determinants of perceptual analysis. Journal of Experimental Psychology: General, 113, 94-111.

GARNER, W. R. (1970), The stimulus in information processing. American Psychologist, 25, 350-358.

GARNER, W. R. (1974). The processing of information and structure. Potomac, MD: Erlbaum.

GARNER, W. R. (1976). Interaction of stimulus dimensions in concept and choice processes. Cognitive Psychology, 8, 98-123.

GATI, I., \& TVERSKY, A. (1982). Representations of qualitative and quantitative dimensions. Journal of Experimental Psychology, 8, 325-340.

GiBson, E. J. (1969). Principles of perceptual learning and development. New York: Appleton-Century-Crofts.

HANDEL, S., \& IMAI, S. (1972). The free classification of analyzable and unanalyzable stimuli. Perception \& Psychophysics, 12, 108-116.

HeLm, C. E. (1964). Multidimensional ratio scaling analysis of perceived color relations. Journal of the Optical Society of America, 54, 252-262.

HYMAN, R., \& WELL, A. (1967). Judgments of similarity and spatial models. Perception \& Psychophysics, 2, 233-248.

Hyman, R., \& Well, A. (1968). Perceptual separability and spatial models. Perception \& Psychophysics, 3, 161-165.

InDOW, T., \& KanAZAWA, K. (1960). Multidimensional mapping of Munsell colors varying in hue, chroma and value. Journal of $E x$ perimental Psychology, 59, 330-336.

INDOW, T., \& UchizoNo, T. (1960). Multidimensional mapping of Munsell colors varying in hue and chroma. Journal of Experimental Psychology, 59, 321-329.
ITTEN, J. (1970). The elements of color. New York: Van Nostrand Reinhold.

Krantz, D. H. (1972). Visual scaling. In D. Jameson \& L. M. Hurvich (Eds.), Visual Psychophysics. Berlin: Springer-Verlag.

Krantz, D. H., \& TVersky, A. (1975). Similarity of rectangles: An analysis of subjective dimensions. Joumal of Mathematical Psychology, 12, 4-34.

KrUSKaL, J. B. (1964). Multidimensional scaling by optimizing goodness of fit to a nonmetric hypothesis. Psychometrika, 29, 1-27.

Lockhead, G. R. (1972). Processing dimensional stimuli: A note. Psychological Review, 79, 410-419.

LOCKHEAD, G. R. (1979). Holistic versus analytic process models: A reply. Joumal of Experimental Psychology: Human Perception \& Per formance, $5,746-755$

Newhale, S. M. (1939). The ratio method in the review of the Munsell colors. American Journal of Psychology, 52, 394-405.

Newhall, S. M., Nickerson, D., \& JUdD, D. B. (1943). Final report of the O. S. A. subcommittee on the spacing of Munsell colors. Journal of the Optical Society of America, 33, 385-418.

RoNACHER, B. , BAUTz, W. (1985). Human pattern recognition: Individually different strategies in analyzing complex stimuli. Biological Cybernetics, 51, 249-261.

SHEPARD, R. N. (1964). Attention and the metric structure of the stimulus. Journal of Mathematical Psychology, 1, 54-87.

ShePP, B. E., BurNs, B., \& McDonough, D. D. (1980). The relation of stimulus structure to perceptual and cognitive development: Further tests of a separability hypothesis. In F. Wilkening, J. Becker, \& T. Trabasso (Eds.), The integration of information by children. Hillsdale, NJ: Erlbaum.

SMITH, J. D., \& BARon, J. (1981). Individual differences in the classification of stimuli by dimension. Joumal of Experimental Psychology: Human Perception \& Performance, 7, 1132-1145.

SMITH, L. B. , KEMLER, D. G. (1978). Levels of experienced dimensionality in children and adults. Cognitive Psychology, 10, 502-532.

TORGERSON, W. S. (1958). Theory and method of scaling. New York: Wiley.

TversKy, A., \&RANTZ, D. H. (1969). Similarity of schematic faces: A test of interdimensional additivity. Perception \& Psychophysics, $5,124-128$.

TVersKy, A., \& Krantz, D. H. (1970). The dimensional representation and the metric structure of similarity data. Journal of Mathematical Psychology, 7, 572-596.

WENDER, K. (1971). A test of independence of dimensions in multidimensional scaling. Perception \& Psychophysics, 10, 30-32.

Wiener-EhrLich, W. K. (1978). Dimensional and metric structures in multidimensional stimuli. Perception \& Psychophysics, 24, 399-414.

WriGHT, H. (1965). Precision of color differences derived from a multidimensional scaling experiment. Journal of the Optical Society of America, 55, 1650-1655.

Wyszecki, G, \& Stiles, W. S. (1982). Color science: Concepts and methods, quantitative data and formulae. New York: Wiley.

YAGER, D., \& TAYLOR, E. (1970). Experimental measures and theoretical account of hue scaling as a function of luminance. Perception \& Psychophysics, 7, 360-364.

\section{NOTES}

1. As is traditional in this research area, we have employed the terms hue, brightmess, and saturation to refer to the psychological dimensions of color. As described by Wyszecki and Stiles (1982), the technically correct terms are hue, lightness, and chroma.

(Manuscript received June 29, 1987; revision accepted for publication October 7,1987 .) 